\title{
Selective killing of cancer cells by ultrasound assisted by biopolymer-coated metal nanoparticles
}

\author{
Y.A. Dyakov ${ }^{1,2 *}$, O.K. Kosheleva ${ }^{1,2}$, T.C. Lai, M. Hsiao ${ }^{1}$, N.G. Chen ${ }^{3}$, C.H. Chen ${ }^{1 *}$ \\ ${ }^{1}$ Genomics Research Center, Academia Sinica, Taipei, Taiwan \\ ${ }^{2}$ Karpov Institute of Physical Chemistry, Moscow, Russia \\ ${ }^{3}$ Institute of Biomedical Engineering, Department of Electrical and Computer Engineering, College \\ of Electrical and Computer Engineering, National Chiao-Tung University, Hsinchu, 300, Taiwan \\ *e-mail:yuri_dyakov@mail.ru;winschen@gate.sinica.edu.tw
}

Key words: Metal nanoparticles, biopolymer coating of nanoparticles, ultrasound treatment of cancer.

Motivation and Aim: Cancer is a leading cause of death worldwide. Traditional physical methods of cancer treatment, such as radiation therapy, including electromagnetic ionizing radiation, elementary particle ionizing beam radiation (electrons, protons, and neutrons), and non-ionizing radiation (photons, microwaves, and radio waves) have numerous undesirable side effects and cannot provide fully efficient treatment of the decease. The ionizing radiation is intended to be directed only at the tumor. Moreover, ionizing radiation itself may cause DNA mutation in normal cells, causing these cells to become cancerous. Non-ionizing radiation therapies are mainly based on hyperthermia in tumors as a result of the higher sensitivity of tumor cells to heat than their normal counterparts. But these methods are also does not have sufficient selectivity.

Methods and Algorithms: In our work we use high intensity ultrasound treatment enforced by addition of biopolymer-coated various metal nanoparticles in tissue or cell culture. In our study we used gold, silver, palladium, mercury, platinum, bismuth, wolfram, and some other metal nanoparticles covered by biologically inertial specific polymer shells. Results: Combination of ultrasound and metal nanoparticles leads simultaneously to the strengthening of the destructive effect of ultrasound irradiation, and the selective toxicity of nanoparticles for cancer cells. This allows decreasing the power of the ultrasound radiation and expands the irradiated area without damaging the surrounded normal tissues. Proper selection of biopolymer shells, as far as the size and composition of metal cores provide the selective killing of cancer cells in the main tumor and in the metastases without negative impact to the surrounded normal tissues and perceptible side effects. This work is the continuation of previous studies [1,2].

Conclusion: We found that nanoparticle-assisted therapy of cancer by the high intensity ultrasound irradiation significantly increase the efficiency of the treatment, allows reducing the intensity of irradiation, and decreasing the side effects.

Acknowledgements: This work was supported by Ministry of Science and Technology of Taiwan (MOST 102-2113-M-001-002-MY5 and NHRI-Ex107-10603EI).

\section{References}

1. Kosheleva O.K. et al. (2016) Selective killing of cancer cells by nanoparticle-assisted ultrasound. Journal of Nanobiotechnology 14: Art.No. 46.

2. Kosheleva O.K. et al. (2017) Nanoparticle-Assisted Ultrasound for Cancer Therapy. Journal of Biomedical Science and Engineering 10(05):54-58. 